\title{
PERIODIC BOEHMIANS
}

DENNIS NEMZER

Department of Mathematics

California State University, Stanislaus

801 W. Monte Vista Avenue

Turlock, CA 95380

(Received November 14, 1988 and in revised form January 23, 1988)

ABSTRACT. A class of generalized functions, called periodic Boehmians, on the unit circle, is studied. It is shown that the class of Boehmians contain all Beurling distributions. An example of a hyperfunction that is not a Boehmian is given. Some growth conditions on the Fourier coefficients of a Boehmian are given. It is shown that the Boehmians, with a given complete metric topological vector space topology, is not locally bounded.

KEY WORDS AND PHRASES. Boehmians, delta sequence, convolution, Fourier coefficients. 1980 AMS SUBJECT CLASSIFICATION CODE. Primary 44A40, 46F15, 46F99, Secondary 42Al6, $42 \mathrm{~A} 24$.

\section{INTRODUCTION.}

The construction of a class $\beta$ of generalized functions, called Boehmians, was first introduced by J. Mikusinski and P. Mikusinski [1]. This construction, which is purely algebraic, is similar to that of Mikusinski operators [2]. In this paper, we will be concerned with Boehmians on the unit circle, denoted by $B(\Gamma)$, first introduced in [3]. The space of Boehmians is quite general. For example, the Boehmians on the real line (unit circle) contain all Schwartz distributions on the real line (unit circle). In Section 4 it will be shown that $B(\Gamma)$ also contains all Beurling distributions on the unit circle. Growth conditions on the Fourier coefficients of a Boehmi an were given in Theorems 5.14 and 5.15 of [3]. These results will be extended in Theorems 4.2 and 4.4. P. Mikusinsk1 [4] also introduced a convergence, called $\delta$-convergence, on $B$, which is similar to Type 1 convergence on Mikusinski operators [2]. Type 1 convergence is not topological (see Section 3), but $\delta$-convergence can be used to induce a complete invariant metric topology on $B$. It is known that the dual of $B$, on the real line, contains only the trivial continuous linear functional. We will show, in Theorem 3.5, that the dual of $\beta(\Gamma)$ contains many continuous linear functionals, but that $\beta(\Gamma)$ is not locally bounded (Theorem 5.2).

In Section 2, the space $\beta(\Gamma)$ is constructed. Most of the material in Section 3 can be found in [3] and [4], but is presented here for the convenience of the reader. In this section two types of convergence on $B(\Gamma)$ are discussed, the Fourier coefficients and Fourier series of a Boehmian are defined, and it is shown (Theorems 3.7 and 3.8) that every Boehmian is the sum of its Fourler series. In Section 4, 
sufficient conditions on a sequence of complex numbers to be the Fourier coefficients of a Boehmian are given (Theorem 4.2). It is also shown that the Fourier coefficients satisfy a growth condition (Theorem 4.4).

An example.(Example 3) of a hyperfunction that is not a Boehmian is given. Also, this example gives rise to a sequence $\left(\xi_{n}\right)$ of complex numbers, $\xi_{n}=e^{o(|n|)}$ as $|n|+\infty$, such that $\left(\xi_{n}\right)$ is not the Fourier coefficients of a Boehmian, answering a question posed in [3]. In Section 5, by using the results in the previous section, we show that $B(\Gamma)$, endowed with a topology, is not a Banach space. The final section is concerned with some remarks and open problems.

2. NOTATION AND CONSTRUCTION OF B.

The unit circle will be denoted by $\Gamma$. $C(\Gamma)\left(L^{1}(\Gamma)\right)$ is the collection of all continuous (integrable) complex-valued functions on $r . C^{N}(\Gamma)\left(C_{m}^{N}(\Gamma)\right.$ ) will be the collection of sequences of continuous (m times continuously differentiable) complexvalued functions on $r$.

The convolution of $f$ and $g$ in $C(\Gamma)$ is denoted by juxtaposition. Thus,

$$
(f g)(x)=1 / 2 \pi \int_{-\pi}^{\pi} f(x-t) g(t) d t
$$

If $f_{n}, f \in C(T)$ for $n=1,2, \ldots, 1$ im $f_{n}=f$ will mean $\left(f_{n}\right)$ converges uniformly to $f$ on $\Gamma$.

A sequence of continuous real valued functions, $\left(\delta_{f}\right)$, will be called an approximate identity or a delta sequence if the following conditions are satisfied:

(i) for each $j, 1 / 2 \pi \int_{-\pi}^{\pi} \delta_{j}(t) d t=1$.

(1i) for each $j$ and all $t, \delta_{j}(t)>0$.

(iii) Given a neighborhood, $V$, of 1 , there exists a positive integer $N$ such that for all $j>N$, the support of $\delta_{j}$ is contained in $v$.

The collection of delta sequences will be denoted by $\Delta$.

DEFINITION 2.1. Let $A \subset C^{N}(\Gamma) \times \Delta$ be defined by

$$
A=\left\{\left(\left(f_{j}\right),\left(\delta_{j}\right)\right) \text { : for each } 1 \text { and each } j f_{i} \delta_{j}=f_{j} \delta_{f}\right\} \text {. }
$$

Two elements $\left(\left(f_{j}\right),\left(\delta_{j}\right)\right)$ and $\left(\left(g_{j}\right),\left(\sigma_{j}\right)\right)$ of $A$ are sald to be equivalent, denoted by $\left(\left(f_{j}\right),\left(\delta_{j}\right)\right) \sim\left(\left(g_{j}\right),\left(\sigma_{j}\right)\right)$, if for all $i$ and $f f_{1} \sigma_{j}=g_{j} \delta_{1}$. A straightforward calculation shows that ' $\sim$ ' is an equivalence relation on $A$. The equivalence classes will be called periodic Boehmians.

DEFINITION 2.2. The space of periodic Boehmians, denoted by $B$, is defined as:

$$
B=\left\{\left[\left(f_{f}\right) /\left(\delta_{f}\right)\right]:\left(\left(f_{f}\right),\left(\delta_{f}\right)\right) \varepsilon A\right\} \text {. }
$$

For convenience a typical element of $\beta$ will be written as $\left[f_{j} / \delta_{j}\right]$.

By defining a natural addition, multiplication and scalar multiplication on $B$, 1.e. $\left[f_{j} / \delta_{j}\right]+\left[g_{f} / \sigma_{j}\right]=\left[\left(f_{j} \sigma_{j}+g_{j} \delta_{j}\right) / \delta_{j} \sigma_{j}\right],\left[f_{j} / \delta_{j}\right]\left[g_{j} / \sigma_{j}\right]=\left[f_{j} g_{j} / \delta_{j} \sigma_{j}\right]$, and 
$\alpha\left[f_{j} / \delta_{j}\right]=\left[\alpha f_{j} / S_{j}\right]$, where $\alpha$ is a complex number, B becomes an algebra.

Let $\left(\delta_{j}\right) \in C_{\infty}^{N}(\Gamma) \cap \Delta$. Then $D^{\prime}(\Gamma)$ (Schwartz distributions on the unit circle) can be viewed as a subset of $\beta$ by identifying $u$ with $\left[u^{*} \delta_{j} / \delta_{j}\right]$, where $u^{*} \delta_{j}$ denotes the convolution of $u$ and $\delta_{j}$ as distributions (see [5]).

\section{CONVERGENCE AND FOURIER SERIES.}

Let $U$ be a class of sequences on a space $X$ (with or without a topology). We say $x_{n} \underset{U}{\rightarrow} x$ if the sequence $\left(x, x_{1}, x_{2}, \ldots\right)$ is in $U . \quad U$ is called topological if there exists a topology $\tau$ for $X$ such that $x_{n} \stackrel{\bigcup}{\rightarrow} x$ if and only if $x_{n} \stackrel{\tau}{+} x$. A convergence used in Mikusinski operational calculus, called Type 1 convergence, is not topological [6].

In a more general construction of Boehmians, P. Mikusinski [4] defines a convergence, called $\Delta$-convergence, and shows that $\Delta$-convergence is topological. In fact, Mikusinski shows that $B$ is an F-space (a complete topological vector space in which the topology is induced by an invariant metric).

Before we define $\Delta$-convergence, we will define a related convergence, called $\delta$-convergence.

Let $a_{n}$, a $\varepsilon \beta$ for $n=1,2, \ldots$, we say that $\left(a_{n}\right)$ is $\delta$-convergent to a if there exists a delta sequence $\left(\delta_{j}\right)$ such that for each $n$ and $j, a \delta_{j}, a_{n} \delta_{j} \varepsilon C(\Gamma)$, and for each $j \lim a_{n} \delta_{j}=a \delta_{j}$. This will be denoted by $\delta-1 \mathrm{im} a_{n}=a$.

The proof of the next lemma can be found in [4].

LEMMA 3.1. Let $a_{n} \varepsilon \beta, f_{n} \varepsilon C(\Gamma)$ for $n=1,2, \ldots$ If there exists $\left(\delta_{n}\right) \varepsilon \Delta$ such that for each $n$ and $j, a_{n} \delta_{j} \varepsilon C(\Gamma)$ and for each $j, 1 \lim _{n} a_{n} \delta_{j}=f_{j}$, then

$\delta-1$ im $a_{n}=\left[f_{j} / \delta_{j}\right]$.

DEFINITION 3.2. A sequence $\left(a_{n}\right)$ of Boehmians is said to be $\Delta$-convergent to $a$, denoted by $\Delta-1$ im $a_{n}=a$, if there exists a delta sequence $\left(\delta_{n}\right)$ such that for each $n$, $\left(a_{,}-a\right) \delta, \varepsilon C(\Gamma)$ and $\lim \left(a_{n}-a\right) \delta_{n}=0$.

...AKK. A sequence of Boehmians $\left(a_{n}\right)$ is $\Delta$-convergent to $a$ if and only if each subsequence of $\left(a_{n}\right)$ contains a subsequence $\delta$-convergent to $a,[4]$.

The Fourier coefficients of an $L^{1}(\Gamma)$ function are defined in the usual way. That is, if $f \in L^{1}(\Gamma)$ define

$$
C_{k}(f)=1 / 2 \pi \int_{-\pi}^{\pi} f(t) e^{-1 k t} d t \text {, for } k=0, \pm 1, \pm 2, \ldots \text {. }
$$

From the definition of a delta sequence it follows that if $\left(\delta_{n}\right) \varepsilon \Delta$, then for each $k, 11 m_{n} c_{k}\left(\delta_{n}\right)=1$. It is also easy to show that for $a=\left[f_{j} / \delta_{j}\right]$, if for some positive integer $j_{0}$ and some $k_{0} c_{k_{0}}\left(\delta_{j}\right)=0$, then $c_{k_{0}}\left(f_{j_{0}}\right)=0$. These observations together with the fact that if $a=\left[f_{j} / \delta_{j}\right]$, then for each $n$ and $m, f_{n} \delta_{m}=f_{m} \delta_{n}$, make the following definition possible.

DEFINITION 3.3. Let $a=\left[f_{j} / \delta_{j}\right] \in B_{0}$ For $k=0, \pm 1, \pm 2, \ldots$, define $C_{k}(a)=C_{k}(f) / C_{k}\left(\delta_{j}\right)$, where for fixed $k, j$ is the smallest index such that $c_{k}\left(\delta_{j}\right) \neq 0$. 
The proofs of Theorems 3.4 and $3.7 \mathrm{can}$ be found in [3].

THEOREM 3.4. Let $a, a_{n} \varepsilon \beta$, for $n=1,2, \ldots$ Suppose $\delta-1$ im $a_{n}=a$, then for each $k$, $\lim _{n} C_{k}\left(a_{n}\right)=C_{k}(a)$.

In this sequel, unless otherwise stated, the topology for $\beta$ will be the one induced by $\Delta$-convergence. J. Burzyk and T.K. Boehme, separately, have shown that Boehmians on the real line have no continuous linear functionals other than the trivial one. As the next theorem will show this is not the case for periodic Boehmians. In fact, there are enough continuous linear functionals to separate points.

THEOREM 3.5. Let $a_{n}$, a $\varepsilon \beta$, for $n=1,2, \ldots$. If $\Delta-1$ im $a_{n}=a$, then for each $k, \lim _{n} C_{k}\left(a_{n}\right)=C_{k}(a)$.

PROOF. Follows from the remark following Definition 3.2 and Theorem 3.4 .

DEFINITION 3.6. Let a $\varepsilon B$, then the Fourier series of a is $\sum_{k=-\infty}^{\infty} C_{k}(a) e^{1 k t}$.

THEOREM 3.7. For each a $\varepsilon \beta, a=\delta-11 m_{n} \sum_{k=-n}^{n} C_{k}(a) e^{i k t}$.

THEOREM 3.8. For each a $\varepsilon$, a $a=\Delta-1 \mathrm{im}_{n} \sum_{k=-n}^{n} C_{k}(a) e^{i k t}$.

PROOF. Follows from the remark following Definition 3.2 and Theorem 3.7.

4. GROWTH CONDITIONS ON THE FOURIER COEFFICIENTS.

In the construction of Beurling distributions, see Example 2, a real-valued even function $\omega$ defined on the integers $Z$ satisfying the following conditions is required.

(A) $0_{\infty}=\omega(0)<\omega(n+m)<\omega(n)+\omega(m) \quad$ for all $n, m \in Z$.

(B) $\quad \sum_{n=1}^{\infty} \omega(n) / n^{2}<\infty$.

Functions that satisfy conditions (A) and (B) w1ll play an important role in this section.

The proof of the next technical lemma is similar to the proof of Theorem 1.2 .7 in [7].

LEMMA 4.1. Suppose $\omega$ is a real-valued even function satisfying conditions (A) and (B). Let $\omega_{1}(n)=\max \{\omega(m):|m|<|n|\}$. Then $\omega_{1}$ also satisfies conditions (A) and (B). PROOF. It is routine to show that $\omega_{1}$ satisfies condition (A). To show that $\omega_{1}$ satisfies condition (B), partition the set of nonnegative integers $N$ into the sets $F, G$, and $H$ as follows: $F=\left\{n \varepsilon N: \omega(n)=\omega_{1}(n)\right\}$, $G=\left\{n \varepsilon N: \omega(n-1)=\omega_{1}(n-1), \omega(n)<\omega_{1}(n), \omega(n+1)=\omega_{1}(n+1)\right\}$, and $H=N \backslash(F \cup G)$. First, consider the set $H . \quad H=U V_{m}$, where for $m=1,2, \ldots$ there exist $1_{m}, f_{m} \varepsilon N$ with

$i_{m}<j_{m}<1_{m+1}$ such that $v_{m}=\left\{n \in N: \omega(n)<\omega_{1}(n)\right.$ for $n=1_{m}, i_{m}+1, \ldots, j_{m}$, $\left.\left.\omega\left(1_{m}-1\right)=\omega_{1}\left(i_{m}\right), \omega\left(j_{m}+1\right)=\omega_{1}\left(j_{m}+1\right)\right\} \cdot \omega_{1}\left(j_{m}+1\right)\right\}$. Consider a typical $v_{m}$ for which $i_{m}>2$. (If $\omega(n)=\omega_{1}(n)$ for all $n$ or if $j_{m}=\infty$ the lemma is trivial). Let $\ell=\min \left\{i_{m}-1, j_{m}-i_{m}\right\}$. Let $E=\left\{n \in N: i_{m}<n<i_{m}+\ell\right.$ and $\left.\omega(n)<\omega\left(i_{m}-1\right) / 3\right\}$, $E^{\prime}=\left\{n \varepsilon N: i_{m}<n<i_{m}+l\right.$ and $\left.\omega(n)>\omega\left(i_{m}-1\right) / 3\right\}$, and $E^{*}=\left\{n \varepsilon N: n=1_{m}-1+x_{1}-x_{2}>1_{m}, x_{1}, x_{2} \varepsilon E\right\}$ 
Let $|\cdot|=$ cardinality of $\cdot$. It then follows that $E^{\star} \subset E^{\prime}$ and

$\left|E^{*}\right|+1>|E|$, hence $\left|E^{\prime}\right|>\ell / 2$. By. using estimates and considering cases $\left(\ell=i_{m}-1\right.$ or $\left.\ell=j_{m}-i_{m}\right)$, we obtain

$$
\sum_{n=i_{m}}^{j_{m}} \omega_{1}(n) / n^{2}<108 \sum_{n=1}^{j} \omega(n) / n^{2}
$$

Thus, $\quad \sum_{n \in H} w(n) / n^{2}<108 \sum_{n \in H} w(n) / n^{2}$.

Now, for each $n \in G \omega_{1}(n) / n^{2}<\omega(n-1) /(n-1)^{2}$.

Hence, $\quad \sum_{n \in G} u(n) / n^{2}<\sum_{n \in G} w(n-1) /(n-1)^{2}$.

Since for each $n \varepsilon F \omega(n)=\omega_{1}(n)$,

$$
\sum_{n \in F} u_{1}(n) / n^{2}=\sum_{n \in F} w(n) / n^{2}
$$

By combining $(4.1),(4.2)$, and $(4.3)$, we see that $\omega_{1}$ satisfies condition (B). Thus, the theorem is proved.

Before giving sufficient conditions for a sequence of complex numbers to be the Fourier coefficients of a Boehmian (Theorem 4.2), we need a result of Bjorck [7]. Suppose $\omega$ is a real-valued continuous function on $R$ satisfying $0=\omega(0)<\omega(x+y)$

$$
<\omega(x)+\omega(y) \text { for all } x, y \in R, \int_{1}^{\infty} \omega(t) / t^{2}<\infty \text {, and } \omega(t)<\ln (1+|t|) \text {. }
$$

Then given any compact set $K$ of $R$ and any neighborhood $V$ of $K$, there exists a continuous function such that $0<<1$, the support of is contained in $V$, and $\equiv 1$ on K. Mreover, $(t)=0\left(e^{-\omega(t)}\right)$ as $|t| \rightarrow \infty$, where denotes the Fourier transform of

By using the above, we may construct an approximate identity $\left(\delta_{f}\right)$ such that for each $j, C_{n}\left(\delta_{j}\right)=0\left(e^{-\omega(n)}\right)$ as $|n| \rightarrow \infty$.

THEOREM 4.2. Let $\omega$ be a real-valued even function satisfying conditions (A) and (B). Suppose $\left(\xi_{n}\right)$ is a sequence of complex numbers such that $\xi_{n}=0\left(e^{\omega(n)}\right)$ as $|n|+\infty$, then there exists a Boehmian a $\varepsilon$ Bsuch that $C_{n}(a)=\xi_{n}$ for all $n$.

PROOF. Let $\omega^{*}(n)=\omega_{1}(n)+\sqrt{|n|}$, where $\omega_{1}$ is as in Lemma 4.1 . Then $\omega^{*}$ satisfies conditions (A) and (B). Let $\omega^{*}(t)$ be the plecewise linear extension of $\omega^{*}$ to $R$.

Since $\omega^{*}(t)$ is increasing on $[0, \infty)$ and $\sum_{n=1}^{\infty} \omega^{*}(n) / n^{2}<\infty, \int_{1}^{\infty} \omega^{*}(t) / t^{2} d t<\infty$. Also, $\omega^{*}(t)>\ln (1+|t|)$. So there exists $\left(\delta_{j}\right) \varepsilon \Delta$ such that for each $j$, $C_{n}\left(\delta_{j}\right)=0\left(e^{-\omega *(n)}\right)$ as $|n|+\infty$. Let $p_{m}(t)=\sum_{k=-m}^{m} \xi_{k} e^{i k t}$, for $m=0,1,2, \ldots$. Then, for 
each $j$, the sequence $\left(p_{m} \varepsilon_{j}\right)$ converges uniformly. Hence for some a $\varepsilon \beta, \delta-1$ im $p_{m}=a$. Now, for $m>|n| C_{n}\left(p_{m}\right)=\xi_{n}$. Therefore, by Theorem 3.4, for each $n c_{n}(a)=\xi_{n}$. Th1s establishes the theorem.

The following lemma is a special case of Theorem XV from [8].

LEMMA 4.3. Let $\omega(t)$ be an increasing function for $t \geqslant 0$, such that

$$
\int_{1}^{\infty} \omega(t) / t^{2} d t=\infty \text {. Suppose } f \varepsilon L^{1}(\Gamma) \text { such that } C_{-n}(f)=0\left(e^{-\omega(n)}\right) \text { as } n+\infty \text {. If } f
$$

vanishes almost everywhere on any interval, then $f \equiv 0$.

The next theorem shows that the Fourier coefficients of a Boehmian cannot grow too fast.

THEOREM 4.4. Let $\omega: Z \rightarrow R$ be an increasing function for $n=0,1,2, \ldots$ and

$\sum_{n=1}^{\infty} \omega(n) / n^{2}=\infty$. Suppose $\left(\xi_{n}\right)$ is a sequence of complex numbers such that there exist positive A, M, and $\varepsilon$ such that $\left|\xi_{n}\right|>A e^{\varepsilon w(n)}$ for al1 $n>M$. Then ( $\left.\xi_{n}\right)$ is not the Fqurler coefficients of a Boehmian.

PROOF. Suppose $\left|\xi_{n}\right|>A e^{\varepsilon \omega(n)}$ for all $n>M$. Let $p_{n}(t)=\sum_{k=-n}^{n} \xi_{k} e^{i k t}$. Suppose $\left(\delta_{j}\right) \varepsilon \Delta$. Extend $\omega(n)$ linearly to a function $\omega(t)$ on $(0, \infty)$. Now, since $\omega(t)$ is increasing and $\sum_{n=1}^{\infty} \omega(n) / n^{2}=\infty, \int_{1}^{\infty} \omega(t) / t^{2} d t=\infty$. Then by the previous

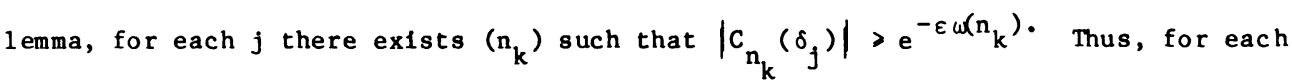
$j, p_{n} \delta_{j}(t)=\sum_{k=-n}^{n} \xi_{k} C_{k}\left(\delta_{j}\right) e^{i k t}$ does not converge and the result follows by Theorem EXAMPLES. 1) If $\omega(n)=\ln (1+|n|)$, then $D^{\prime}(\Gamma)$ (periodic Schwartz distributions [5]) is isomorphic to $\left\{a \varepsilon\right.$ : there exist $\alpha>0$ such that $C_{n}(a)=O\left(e^{\alpha \omega(n)}\right)$ as $|n|+\infty\}$.

2) Let $\omega$ be an even real-valued function on the integers $Z$ which satisfies:

(A) $0=\omega(0)<\omega(n+m)<\omega(n)+\omega(m)$ for all $n, m \varepsilon Z$.

(B) $\quad \sum_{n=1}^{\infty} \omega(n) / n^{2}<\infty$.

(C) There exist real $a$ and positive $b$ such that $\omega(n)>a+b \ln (1+|n|)$ for al 1 neZ.

(D) $\quad \omega$ is concave down on $n=0,1,2, \ldots$.

The set of Beurling distributions on the unit circle $P^{\prime}{ }_{\omega}$ is dual space of $P_{\omega}$ (a test function space with a locally convex topology). $\quad P_{\omega}^{\prime}$ is isomorphic to the collection of all sequences of complex numbers of w-slow growth [9] (a sequence ( $\xi_{n}$ ) of complex numbers is said to be of $\omega$-slow growth if there exist constants $M$ and a such that for all $\left.\mathrm{n} \varepsilon \mathrm{Z},\left|\xi_{\mathrm{n}}\right|<\mathrm{Me}^{\mathrm{a}(\omega(\mathrm{n}))}\right)$. So, by Theorem 4.2, B contains all periodic Beurling distributions. 
By taking $\omega(\mathrm{n})=\mathrm{n}^{\alpha}(\omega(\mathrm{n})=\varepsilon \mathrm{n}, \varepsilon>0)$, where $0<\alpha<1$, in Theorem 4.2 (Theorem 4.4), we obtain Theorem 5.14 (Theorem 5.15) in [3]. The next example shows that Theorem 4.2 (Theorem 4.4) is more general than Theorem 5.14 (Theorem 5.15). 3) For $\alpha \geqslant 1$ let $\omega_{\alpha}(t)=t /(\ln t)^{\alpha}$ for $t>e^{\alpha+1}$, and for $0<t<e^{\alpha+1}$, $\omega_{\alpha}$ is the linear function whose graph passes through the origin and $\left(e^{\alpha+1}, \omega_{\alpha}\left(e^{\alpha+1}\right)\right)$. Define $\omega_{\alpha}$ for $t<0$ by $\omega_{\alpha}(-t)=\omega_{\alpha}(t)$. It is not difficult to show that $\omega_{\alpha}$ satisfies condition (A), since, if $\varphi$ is any concave down increasing function for $t>0$, such that $\varphi(0)=0$ and $\varphi(-t)=\varphi(t)$, then $\varphi$ satisfies condition (A). Now, let $\omega_{\alpha}(n)$ denote the restriction of $\omega_{\alpha}(t)$ to the integers. Thus, by Theorem 4.2, $\left(\exp \left(\omega_{\alpha}(n)\right)\right)$ is the Fourier coefficients of a Boehmian when $\alpha>1$; but $\left(\exp \left(\omega_{1}(n)\right)\right)$, by Theorem 4.4, is not the Fourier coefficients of a Boehmian.

The above answers one (and partially another) question posed in [3]. Not every sequence $\left(\xi_{n}\right), \xi_{n}=e^{0(|n|)}$ as $|n|+\infty$, is the sequence of Fourier coefficients for a Boehmian. Secondly, "How does $\beta$ compare with the set of hyperfunctions?" Since $\limsup _{n}\left|\exp \left(\omega_{1}(n)\right)\right|^{1 / n}=1,\left(\exp \left(\omega_{1}(n)\right)\right)$ is the Fourier coefficients of a hyperfunction [10], but is not the Fourier coefficients of a Boehmian.

.5. TOPOLOGY OF $B$.

A topological vector space $X$ is called a topological algebra if it is an algebra over the real or complex numbers and multiplication is continuous in each argument separately. It is not difficult to show that $B$ endowed with $\Delta$-convergence is a topological algebra.

DEFINITION 5.1. Let $X$ be an F-algebra (F-space which is an algebra over the real or complex numbers) with metric $d$. The $X$ is called a p-normed algebra if there is a function $\|\cdot\|: X \rightarrow R$ such that:

(1) for each $x, y \in x, d(x, y)=\| x-y||$.

(ii) for each $x, y \in x,\|x y\|<\|x\|\|y\|$.

(iii) there exists $0<p<1$ such that for all scalars $\alpha$ and each $x \varepsilon X$ ||$\alpha x||=|\alpha|^{p}|| x||$.

Zelazko [11] has proved that a complete metric topological algebra 1s locally bounded if and only if it is a p-normed algebra.

THEOREM 5.2. B is not locally bounded.

PROOF. Suppose $B$ is locally bounded. Since $B$ is a complete topological algebra, there exists an equivalent $p$-norm $\|\cdot\|$ on $\beta$. Now for $n=0,1,2, \ldots$,

let $a_{n}=\sum_{\ell=0}^{n} \alpha_{\ell} s^{\ell}$, where $s^{\ell}=\left[\delta_{j}(\ell) / \delta_{j}\right]$ for $\ell=0,1,2, \ldots, n$, $\left(\delta_{j}\right) \varepsilon C_{\infty}^{N}(\Gamma) \cap \Delta$, and $\alpha_{\ell}=1 /\left(1^{\ell} \ell !\right)$ for $\ell=0,1,2, \ldots, n$. Given $\varepsilon>0$ there exists a positive integer $M$ such that for $n>m>M$,

$$
\left\|a_{n}-a_{m}\right\|=\left\|\sum_{\ell=m+1}^{n} \alpha_{\ell} s^{\ell}\right\| \leqslant\left.\sum_{\ell=m+1}^{n}|1 / \ell !| p|| s\right|^{\ell}<\varepsilon .
$$

That is, $\left(a_{n}\right)$ is a Cauchy sequence and since $\beta$ is complete, there exists an a $\varepsilon$ such that $a=\Delta-1 \operatorname{lm}_{n} \sum_{\ell=0}^{n} \alpha_{\ell} s^{\ell}$. So, by Theorem 3.5 , for each $k$ 
$C_{k}(a)=\sum_{\ell=0}^{\infty}(\pi k)^{\ell} / \ell !=e^{\pi k}$, which is impossible by Theorem 4.4. Hence $\beta$ must not be locally bounded.

COROLLARY 5.3. $\quad B$ is not normable.

6. SOME CONCLUDING REMARKS AND QUESTIONS.

(1) It can be shown that if the sequence $\left(\xi_{n}\right)$ is the Fourier coefficients of a Boehmian a that satisfies the hypothesis of Theorem 4.2 (for some function $\omega$ ), then 1 imsup $n\left|\xi_{n}\right|^{1 / n}<1$ and hence a is a hyperfunction [10]. Example 3 presents a hyperfunction that is not a Boehmian. Is $B$ properly contained in the set of hyperfinn-วas?

(2) Theorem 4.2 gives sufficient conditions for a sequence to be the Fourier coefficients of a Bohmian. Are these conditions also necessary?

(3) $B$ with $\Delta$-convergence is an F-space [4]. Theorem 3.5 shows that $B$ has enough continuous linear functionals to separate points, but Corollary 5.3 shows that $B$ is not a Banach space. Is $B$ with $\Delta$-convergence a Frechet space?

(4) What about boundary-value theory? That is, what type of harmonic (analytic) function in the unit disk has a Boehmian for its boundary value? Much mathematical research has been centered around boundary-value problems of harmonic (analytic) functions in the unit disk; see for example [9] and [10].

\section{REFERENCES}

1. MIKUSINSKI, J. and MIKUSINSKI, P., Quotients de suites et leurs applications dans 1'analyse fonctionnelle, Comptes Rendus, 293, serie 1 (1981), 463-464.

2. MIKUSINSKI, J., Operational Calculus, Pergamon Press, New York, 1959.

3. NEMZER, D., Periodic Generalized Functions, Rocky Mtn. J. Math., (to appear).

4. MIKUSINSKI, P., Convergence of Boehmians, Japan J. Math. 9 (1983), 159-179.

5. SCHWARTZ, L., Theorie des distributions, Herman, Paris, 1966.

6. BOEHME, T.K., On Mikusinski operators, Studia Math., 1969, 127-140.

7. BJORCK, G., Linear partial differential operators and generalized distributions, Ark. Mat. 6 (1966), 351-407.

8. LEVINSON, N., Gap and density theorems, American Math. Soc., New York, 1940.

9. Collier, M.G. and Kelingos, J.A., Periodic Beurling Distributions, Acta. Math. Hung. 42 (1983), 261-278.

10. JOHNSON, G., Harmonic functions on the unit disk, Illinois J. Math. 12, (1968), 366-385.

11. ZELAZKO, W., On the locally bounded and m-convex topological algebras, Studia Math., (1960), 333-356. 


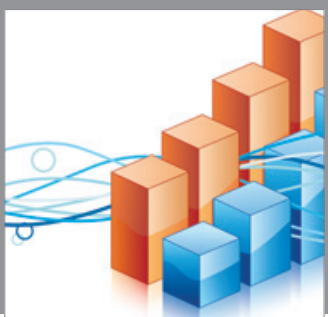

Advances in

Operations Research

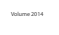

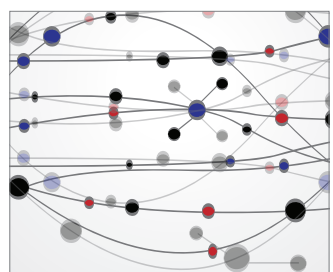

\section{The Scientific} World Journal
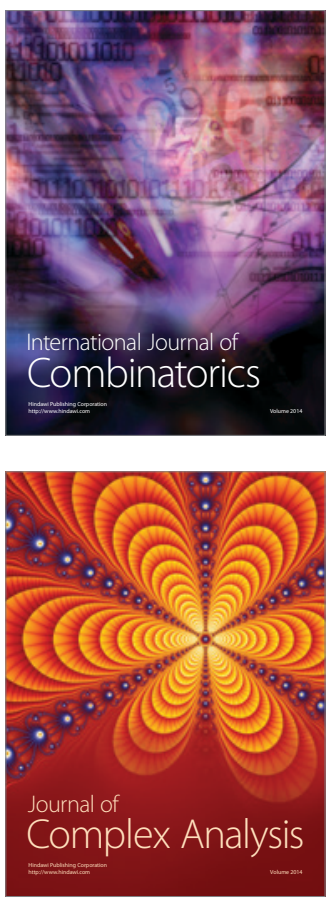

International Journal of

Mathematics and

Mathematical

Sciences
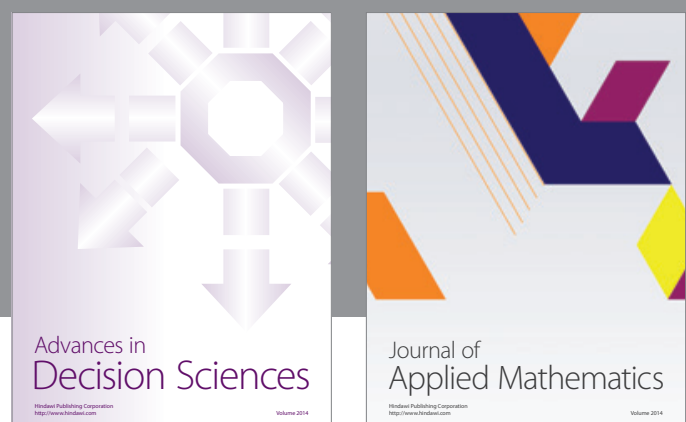

Journal of

Applied Mathematics
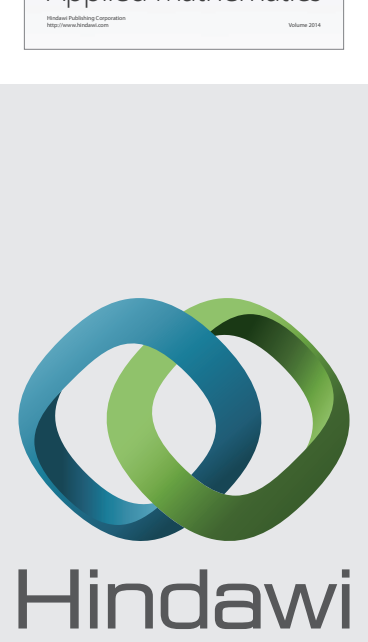

Submit your manuscripts at http://www.hindawi.com
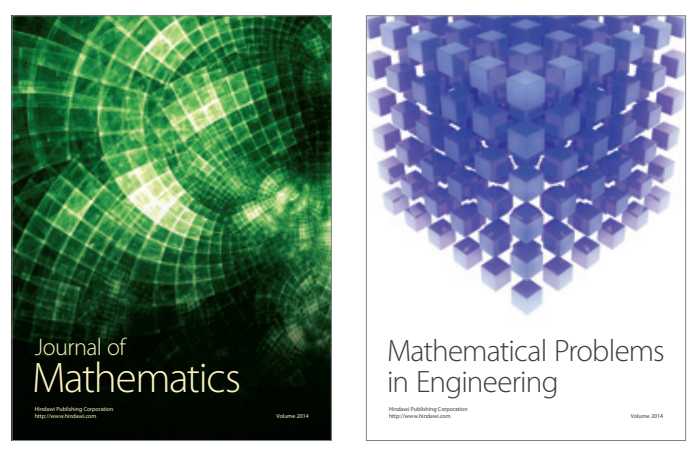

Mathematical Problems in Engineering
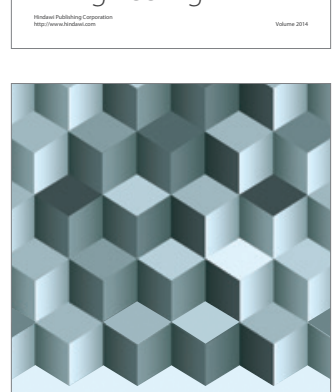

Journal of

Function Spaces
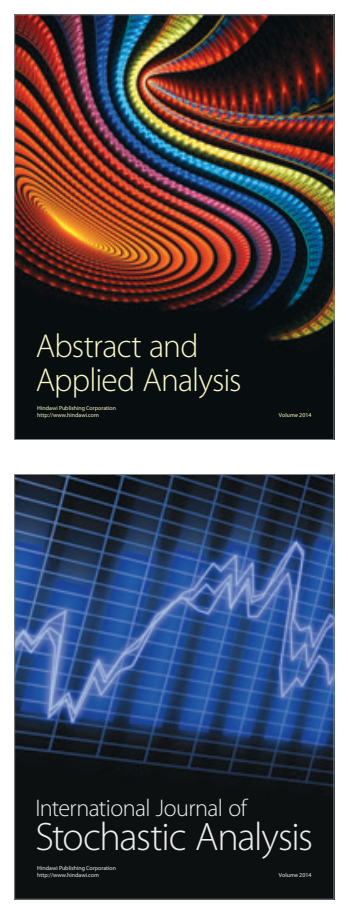

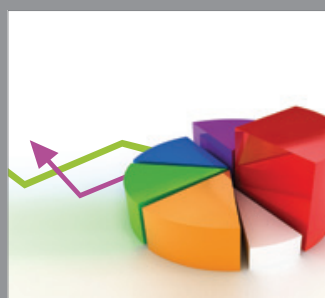

ournal of

Probability and Statistics

Promensencen
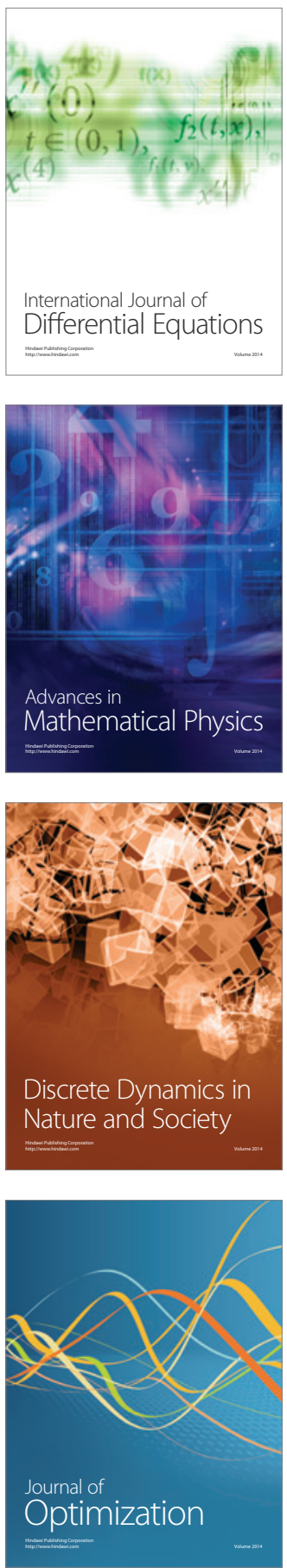